\title{
Grid refinement in the three-dimensional hybrid recursive regularized lattice Boltzmann method for compressible aerodynamics
}

\author{
Y. Feng $\odot, ~ S . ~ G u o,{ }^{*}$ J. Jacob, and P. Sagaut \\ Aix Marseille Univ, CNRS, Centrale Marseille, M2P2, Marseille, France
}

\begin{abstract}
Grid refinement techniques are of paramount importance for computational fluid dynamics approaches relying on the use of Cartesian grids. This is especially true of solvers dedicated to aerodynamics, in which the capture of thin shear layers require the use of small cells. In this paper, a three-dimensional grid refinement technique is developed within the framework of hybrid recursive regularized lattice Boltzmann method (HRR-LBM) for compressible high-speed flows, which is an efficient collide-stream-type method on a compact D3Q19 stencil. The proposed method is successfully assessed considering several test cases, namely, an isentropic vortex propagating through transition interface, shock-vortex interaction with intersection between grid refinement interface and shock corrugation, and transonic flows over three-dimensional DLR-M6 wing with seven levels of grid refinement.
\end{abstract}

\section{INTRODUCTION}

The lattice Boltzmann method (LBM) is an approach to simulate fluid flows based on the discretization in space and velocity of the Boltzmann equation [1-3]. Due to its advantages for massively parallel computing as well as its ability to handle very complex geometries, LBM has gained popularity as a promising approach for computational fluid dynamics. From initial academic benchmarks [4,5], the LB methods was quickly extended to large scale applications and spread towards exascale applications: full-scale vehicles [6], large-scale fuel cells [7], urban scale environment flows $[8,9]$, meteorological flows [10], and complex hydrodynamic problems [11], often with outstanding results.

Classical LB methods rely on the use of uniform grids along with the collide-stream algorithm. Therefore, the numerical cost simultaneously increases in both space and time. As a consequence, the total memory cost and the computational time increase extremely fast. As reported in Refs. [12,13], the capacity of LB method using uniform grids is reaching up to a trillion nodes on current petascale supercomputers. To reduce the numerical cost in large scale simulations, grid refinement techniques has been introduced into the LBM framework [14-24].

A pioneering work of a node-based grid refinement concept was conducted by Filipova and Hänel [14], in which fine grid node values (either colocated with coarse grid nodes or are located at mid-point of two coarse grid points) and the nonequilibrium distribution functions are rescaled between fine grid and coarse grid. This approach has been further developed and investigated by a number of works [15,16,18,19,25-29]. Unlike the node-based approach, a cellbased grid refinement was proposed by Chen et al. [22], which

\footnotetext{
*shaolong.guo@univ-amu.fr
}

aims to guarantee satisfaction of conservation laws between different space resolutions. The approach has been studied and extended in several works $[12,13,17,30,31]$. Nevertheless, most of the grid refinement techniques of LB methods were successfully developed on standard lattices (D2Q9, D3Q19) for nearly incompressible and athermal flows [25-29,32-34].

The classical collision-streaming procedure of LB method offers many degrees of freedom to improve the collision models. However, nonphysical discontinuous profiles, lack of conservation for some physical quantities, under-resolved scales and varying relaxation time at the grid refinement interfaces may induce different numerical stability and accuracy issues in LB methods depending on the collision models. Thanks to their capability to damp nonhydrodynamic modes and their clear physical image, the regularized collision model and its variants present good robustness and accuracy on both uniform grid and multi-resolution grid [23,35]. The connection and comparison of regularized model with SRT, MRT, entropic, and central moment models are reported in Refs. [35-40]. It is worth reminding that regularized collisions models can be rewritten as two-relaxation-time models, and also as a particular case of entropic models when considering a global entropy instead of a local one.

The reason why grid refinement for compressible LB methods has been addressed by very few authors only is that most existing LB models for high Mach flows rely on multispeed lattices with wide stencils (D2Q17, D3Q39, D3Q343) that render the design of grid interface conditions much more difficult [41-44]. A grid refinement technique was proposed on compressible LBM in multispeed type [44]. The D3Q343 lattice was adopted in their model for which the rescaling procedure needs to be performed on multiple layer interface nodes [e.g., $7 \times 7 \times(3+2+1)$ nodes may be involved in one dimensional domain decomposition]. Accordingly, it dramatically increases the algorithm complexity and numerical cost in three dimensional simulations. Its high numerical costs 
motivated the development of alternative models and related grid refinement technique. Recently, some LB models based on the nearest-neighbor lattices for high Mach flows were proposed [45-49]. Accurately propagating acoustic wave through grid transition interface is recognized as a major issue in grid refinement algorithm for compressible flows, and the difficulties are strengthened in compressible LBM for aerodynamics. Given the promising results obtained for the simulation of compressible flows in high subsonic to supersonic regimes with the hybrid recursive regularized LB model (HRR-LBM) on D2Q9 and D3Q19 lattices [46,48,49], the development of grid refinement techniques for compressible LBM on standard lattices is regarded as a key step towards the development of an efficient tool for compressible aerodynamics.

The objective of this paper is to propose a grid refinement algorithm for compressible aerodynamics relying on the D3Q19 lattice model based on HRR-LBM. It is organized as follows: Sec. II describes the key elements of the hybrid recursive regularized lattice Boltzmann model for compressible flows; in Sec. III, three-dimensional grid refinement for both flow field solved by LBM and entropy equation by finite volume method is developed and the implementation is described in details. Then, the results of three classical numerical tests are illustrated and discussed in Sec. IV. Finally, Sec. V draws conclusions.

\section{HRR LATTICE BOLTZMANN MODEL FOR COMPRESSIBLE FLOWS}

\section{A. Macroscopic governing equations}

In the hybrid recursive regularized LB method defined in Refs. $[46,48,49]$, the associated macroscopic equations are the compressible Navier-Stokes equations for mass and momentum conservation supplemented by an entropy equation written in nonconservative form, leading to

$$
\begin{gathered}
\frac{\partial \rho}{\partial t}+\frac{\partial}{\partial x_{\alpha}}\left(\rho u_{\alpha}\right)=0, \\
\frac{\partial}{\partial t}\left(\rho u_{\alpha}\right)+\frac{\partial}{\partial x_{\beta}}\left(\rho u_{\alpha} u_{\beta}\right)=-\frac{\partial}{\partial x_{\alpha}} p+\frac{\partial}{\partial x_{\beta}} \Pi_{\alpha \beta}, \\
\frac{\partial s}{\partial t}+u_{\alpha} \frac{\partial s}{\partial x_{\alpha}}=\frac{1}{\rho T} \frac{\partial}{\partial x_{\alpha}}\left(\lambda \frac{\partial T}{\partial x_{\alpha}}\right)+\frac{1}{\rho T} \Pi_{\alpha \beta} \frac{\partial u_{\alpha}}{\partial x_{\beta}},
\end{gathered}
$$

where $\rho, u, p, s$, and $T$ are the density, velocity, pressure, entropy, and temperature, respectively. The thermodynamic closure and the viscous stress $\Pi_{\alpha \beta}$ are given as

$$
\begin{gathered}
s=c_{v} \ln \frac{p}{\rho^{\gamma}}, p=\rho R T, \\
\Pi_{\alpha \beta}=\mu\left[\frac{\partial u_{\beta}}{\partial x_{\alpha}}+\frac{\partial u_{\alpha}}{\partial x_{\beta}}-\frac{2}{3} \frac{\partial u_{\gamma}}{\partial x_{\gamma}} \delta_{\alpha \beta}\right],
\end{gathered}
$$

where $c_{v}, \gamma$, and $R$ are specific heat capacity at constant volume, specific heat ratio, and gas constant, respectively. $\mu$ is the fluid dynamic viscosity, $\lambda$ heat conductivity, and $\delta_{\alpha \beta}$ Kronecker $\delta$.

\section{B. Hybrid recursive regularized $L B$}

In the hybrid approach used in the present paper, the lattice Boltzmann solver aims at solving the mass and momentum conservation Eqs. (1) and (1 b) while a finite volume solver is used for the entropy Eq. (1).

Therefore, evolution equations are solved for $f_{i}\left(x_{\alpha}, t\right)$, the density distribution of particles with discrete velocity $c_{i \alpha}$ at $\left(x_{\alpha}, t\right)$, which can be obtained at time $t+\delta_{t}$ via a collidestream algorithm $[4,41,50,51]$ that can be interpreted as Strang splitting method [52]. The compressible lattice Boltzmann BGK algorithm with hybrid recursive regularization is expressed as

$$
\begin{aligned}
& \text { collision : } f_{i}^{\prime}\left(x_{\alpha}, t\right)=f_{i}^{\mathrm{eq}}\left(x_{\alpha}, t\right)+\left(1-\frac{1}{\bar{\tau}}\right) \mathcal{R}\left(f_{i}^{\mathrm{neq}}\right) \\
& +\frac{\delta_{t}}{2} \psi_{i}\left(x_{\alpha}, t\right), \\
& \text { streaming : } f_{i}\left(x_{\alpha}, t+\delta_{t}\right)=f_{i}^{\prime}\left(x_{\alpha}-c_{i \alpha} \delta_{t}, t\right) \text {, }
\end{aligned}
$$

where $f_{i}^{\prime}$ denotes density distribution after collision. $\bar{\tau}$ the nondimensional relaxation time which is related with dynamic viscosity through $\mu=p(\bar{\tau}-0.5) \delta_{t} . \mathcal{R}\left(f_{i}^{\text {neq }}\right)$ denotes the hybrid recursive regularization on off-equilibrium distribution function $f_{i}^{\text {neq }}=f_{i}-f_{i}^{\text {eq }}+\delta_{t} \psi_{i} / 2$. Then, the macroscopic density and momentum incorporated the general forcing term $\psi_{i}$ are updated as

$$
\begin{aligned}
\rho & =\sum_{i} f_{i}, \\
\rho u_{\alpha} & =\sum_{i} c_{i \alpha} f_{i}+\frac{\delta_{t}}{2} \sum_{i} c_{i \alpha} \psi_{i} .
\end{aligned}
$$

A classical three-dimensional lattice with 19 discrete velocities $(D 3 Q 19)$ is used in this work. The discrete velocities $c_{i \alpha}$ is given by

$$
\begin{cases}(0,0,0) & i=0 \\ ( \pm 1,0,0),(0, \pm 1,0),(0,0, \pm 1) & i=1-6 \\ ( \pm 1, \pm 1,0),( \pm 1,0, \pm 1),(0, \pm 1, \pm 1) & i=7-18\end{cases}
$$

The improved third-order equilibrium distribution function on D3Q19 lattice is used as $f^{\text {eq }}$ in Eq. (5), which is given by [49]

$$
\begin{aligned}
f_{i}^{\mathrm{eq}}= & w_{i}\left\{\rho+\frac{c_{i \alpha} \rho u_{\alpha}}{c_{s}^{2}}+\frac{\mathcal{H}_{i, \alpha \beta} \mathcal{A}_{\alpha \beta}^{(0)}}{2 c_{s}^{4}}+\frac{1}{6 c_{s}^{6}}\right. \\
& \times\left[3\left(\mathcal{H}_{i, x x y}+\mathcal{H}_{i, y z z}\right)\left(\mathcal{A}_{x x y}^{(0)}+\mathcal{A}_{y z z}^{(0)}\right)\right. \\
& +\left(\mathcal{H}_{i, x x y}-\mathcal{H}_{i, y z z}\right)\left(\mathcal{A}_{x x y}^{(0)}-\mathcal{A}_{y z z}^{(0)}\right) \\
& +3\left(\mathcal{H}_{i, x z z}+\mathcal{H}_{i, x y y}\right)\left(\mathcal{A}_{x z z}^{0)}+\mathcal{A}_{x y y}^{(0)}\right) \\
& +\left(\mathcal{H}_{i, x z z}-\mathcal{H}_{i, x y y}\right)\left(\mathcal{A}_{x z z}^{(0)}-\mathcal{A}_{x y y}^{(0)}\right) \\
& +3\left(\mathcal{H}_{i, y y z}+\mathcal{H}_{i, x x z}\right)\left(\mathcal{A}_{y y z}^{(0)}+\mathcal{A}_{x x z}^{(0)}\right) \\
& \left.\left.+\left(\mathcal{H}_{i, y y z}-\mathcal{H}_{i, x x z}\right)\left(\mathcal{A}_{y y z}^{(0)}-\mathcal{A}_{x x z}^{(0)}\right)\right]\right\},
\end{aligned}
$$

where $\mathcal{H}_{i, \alpha \beta}=c_{i \alpha} c_{i \beta}-c_{s}^{2} \delta_{\alpha \beta}$ and $\mathcal{H}_{i, \alpha \beta \gamma}=c_{i \alpha} c_{i \beta} c_{i \gamma}-c_{s}^{2}[c \delta]_{\alpha \beta \gamma}$ denote the second and third order Hermite polynomials, respectively, with $\left[c_{i} \delta\right]_{\alpha \beta \gamma}=c_{i \alpha} \delta_{\beta \gamma}+c_{i \beta} \delta_{\alpha \gamma}+c_{i \gamma} \delta_{\alpha \beta}$. 
$\mathcal{A}_{\alpha \beta}^{(0)}=\rho u_{\alpha} u_{\beta}+\rho c_{s}^{2}(\theta-1) \delta_{\alpha \beta} \quad$ and $\quad \mathcal{A}_{\alpha \beta \gamma}^{(0)}=\rho u_{\alpha} u_{\beta} u_{\gamma}+$ $\rho c_{s}^{2}(\theta-1)[u \delta]_{\alpha \beta \gamma}$ are, respectively, the second- and third-order coefficient of Hermite polynomials with $[u \delta]_{\alpha \beta \gamma}=u_{\alpha} \delta_{\beta \gamma}+u_{\beta} \delta_{\alpha \gamma}+u_{\gamma} \delta_{\alpha \beta}$. The nondimensional temperature $\theta=R T / c_{s}^{2}$ satisfies the equation of state for perfect gas given by $p=\rho c_{s}^{2} \theta$ with $c_{s}$ being lattice sound speed. The weighting factors $w_{i}$ for each discrete velocity are

$$
w_{i}=\left\{\begin{aligned}
\frac{1}{3} & & i=0 \\
\frac{1}{18} & & i=1-6 \\
\frac{1}{36} & & i=7-18
\end{aligned}\right.
$$

In the HRR collision model, the nonphysical modes [53] are filtered by a hybrid recursive regularization operator, which is expressed as

$$
\begin{aligned}
\mathcal{R}\left(f_{i}^{n e q}\right)= & w_{i}\left\{\frac{\mathcal{H}_{i, \alpha \beta} \mathcal{A}_{\alpha \beta}^{(1)}}{2 c_{s}^{4}}+\frac{1}{6 c_{s}^{6}}\right. \\
& \times\left[3\left(\mathcal{H}_{i, x x y}+\mathcal{H}_{i, y z z}\right)\left(\mathcal{A}_{x x y}^{(1)}+\mathcal{A}_{y z z}^{(1)}\right)\right. \\
& +\left(\mathcal{H}_{i, x x y}-\mathcal{H}_{i, y z z}\right)\left(\mathcal{A}_{x x y}^{(1)}-\mathcal{A}_{y z z}^{(1)}\right) \\
& +3\left(\mathcal{H}_{i, x z z}+\mathcal{H}_{i, x y y}\right)\left(\mathcal{A}_{x z z}^{(1)}+\mathcal{A}_{x y y}^{(1)}\right) \\
& +\left(\mathcal{H}_{i, x z z}-\mathcal{H}_{i, x y y}\right)\left(\mathcal{A}_{x z z}^{(1)}-\mathcal{A}_{x y y}^{(1)}\right) \\
& +3\left(\mathcal{H}_{i, y y z}+\mathcal{H}_{i, x x z}\right)\left(\mathcal{A}_{y y z}^{(1)}+\mathcal{A}_{x x z}^{(1)}\right) \\
& \left.\left.+\left(\mathcal{H}_{i, y y z}-\mathcal{H}_{i, x x z}\right)\left(\mathcal{A}_{y y z}^{(1)}-\mathcal{A}_{x x z}^{(1)}\right)\right]\right\},
\end{aligned}
$$

where $\mathcal{A}_{\alpha \beta}^{(1)}=\sum_{i} c_{i \alpha} c_{i \beta} f_{i}^{\text {neq }}$ is the second-order offequilibrium moment and the third-order off-equilibrium moment is recursively computed by using $\mathcal{A}_{\alpha \beta \gamma}^{(1)}=u_{\alpha} \mathcal{A}_{\beta \gamma}^{(1)}+$ $u_{\beta} \mathcal{A}_{\gamma \alpha}^{(1)}+u_{\gamma} \mathcal{A}_{\alpha \beta}^{(1)}$. In addition, the off-equilibrium moment is fractionally approximated by its solution in Chapman-Enskog analysis by using $\mathcal{A}_{\alpha \beta}^{(1, \mathrm{HRR})}=\sigma \mathcal{A}_{\alpha \beta}^{(1)}+(1-\sigma) \mathcal{A}_{\alpha \beta}^{(1, \mathrm{FD})}$, where $\mathcal{A}_{\alpha \beta}^{(1, \mathrm{FD})}$ is given as

$$
\mathcal{A}_{\alpha \beta}^{(1, \mathrm{FD})} \approx-\delta_{t} \bar{\tau} p\left[\frac{\partial u_{\beta}}{\partial x_{\alpha}}+\frac{\partial u_{\alpha}}{\partial x_{\beta}}-\frac{2}{3} \frac{\partial u_{\gamma}}{\partial x_{\gamma}} \delta_{\alpha \beta}\right] .
$$

The second-order isotropic central difference scheme is employed to compute the numerical gradient operator. Then, $\mathcal{A}_{\alpha \beta}^{(1, \mathrm{HRR})}$ is employed in the hybrid recursive regularization Eq. (5).

In addition, a correction term introduced in the forcing term to balance symmetry-breaking errors due to the topology of the lattice and the expression of the collision kernel. It is expressed as [46,49]

$$
\begin{aligned}
\psi_{i}= & \frac{w_{i}}{2 c_{s}^{4}}\left\{\mathcal{H}_{i, x x} \frac{\partial}{\partial x}\left[\rho u_{x}\left(1-\theta-u_{x}^{2}\right)\right]-\mathcal{H}_{i, y z} \frac{\partial}{\partial x}\left(\rho u_{x} u_{y} u_{z}\right)\right. \\
& +\mathcal{H}_{i, y y} \frac{\partial}{\partial y}\left[\rho u_{y}\left(1-\theta-u_{y}^{2}\right)\right]-\mathcal{H}_{i, x z} \frac{\partial}{\partial y}\left(\rho u_{x} u_{y} u_{z}\right) \\
& \left.+\mathcal{H}_{i, z z} \frac{\partial}{\partial z}\left[\rho u_{z}\left(1-\theta-u_{z}^{2}\right)\right]-\mathcal{H}_{i, x y} \frac{\partial}{\partial z}\left(\rho u_{x} u_{y} u_{z}\right)\right\} .
\end{aligned}
$$

\section{Finite volume solver for entropy conservation equation}

As mentioned in Sec. II A, the nonconservative form of entropy conservation Eq. (1) is solved by a finite volume method [46]. The first-order explicit Euler scheme is adopted for time integration, i.e.,

$$
s^{n+1}=s^{n}+\delta_{t}\left[\operatorname{RHS}_{C}\left(s^{n}\right)+\operatorname{RHS}_{D}\left(s^{n}\right)+\Psi^{n}\right],
$$

where $\mathrm{RHS}_{C}, \mathrm{RHS}_{D}$ are related to the convection term and the diffusion term, respectively. $\Psi$ is a source term related to the heat generation induced by viscous dissipation. The convective flux is constructed using MUSCL scheme [54] equipped with the van Albada limiter [55]. The classical second-order central difference scheme is adopted for the diffusion term.

\section{Shock capturing, initial and boundary conditions}

For discontinuous compressible flows, the shock sensor and associated artificial viscosity used in Jameson-SchmidtTurkel (JST) scheme [56] are adopted in the HRR-LB model to handle the shock waves. Accordingly, an effective relaxation time can be summed as $\tau_{e}=\left(\mu+\mu_{s w}\right) / p$ with $\mu$ and $\mu_{s w}$ being fluid dynamic and Jameson's artificial viscosity, respectively. Then the nondimensional relaxation time $\bar{\tau}=$ $\tau_{e} / \delta_{t}+0.5$ is actually employed in the following test cases with shocks. In the following simulations, the inviscid flows are treated as quasi-inviscid, with a very small nondimensional viscosity $\mu=10^{-15}$.

The boundary conditions for distribution functions are implemented by a finite difference-based reconstruction approach along with the hybrid recursive regularization procedure $[35,46]$. First, the macroscopic values $\rho, u, T$, and $s$ on the boundary nodes are prescribed for plane solid walls or estimated for curved solid walls by a cut-cell-based method [57]. For far-field open boundaries, the characteristic boundary conditions coupled with an absorbing sponge layer is used to compute macroscopic values on the boundary nodes. Then the distribution functions are computed as $f_{i}=f_{i}^{\text {eq }}+f_{i}^{\text {neq }}$. The off-equilibrium distribution function $f_{i}^{\text {neq }}$ is recursively reconstructed from macroscopic variables and their gradients. The detailed implementation of boundary conditions is given in Ref. [57].

The initial conditions are also implemented by the reconstruction approach where the distribution functions are summation of $f_{i}^{e q}$ and $f_{i}^{\text {neq }}$. The equilibrium part is directly computed by Eq. (8) while the off-equilibrium distribution function $f_{i}^{\text {neq }}$ is reconstructed from macroscopic variables.

\section{3D GRID REFINEMENT FOR COMPRESSIBLE HRR-LBM}

\section{A. Grid refinement procedure}

In this section, a procedure for three-dimensional grid refinement in the HRR-LBM for compressible flows is proposed. The present grid refinement method is based on multidomain decomposition in which the computational domain is discretized in space with a hierarchy of uniform embedded meshes with a grid step ratio of 2 between two successive refinement areas. 


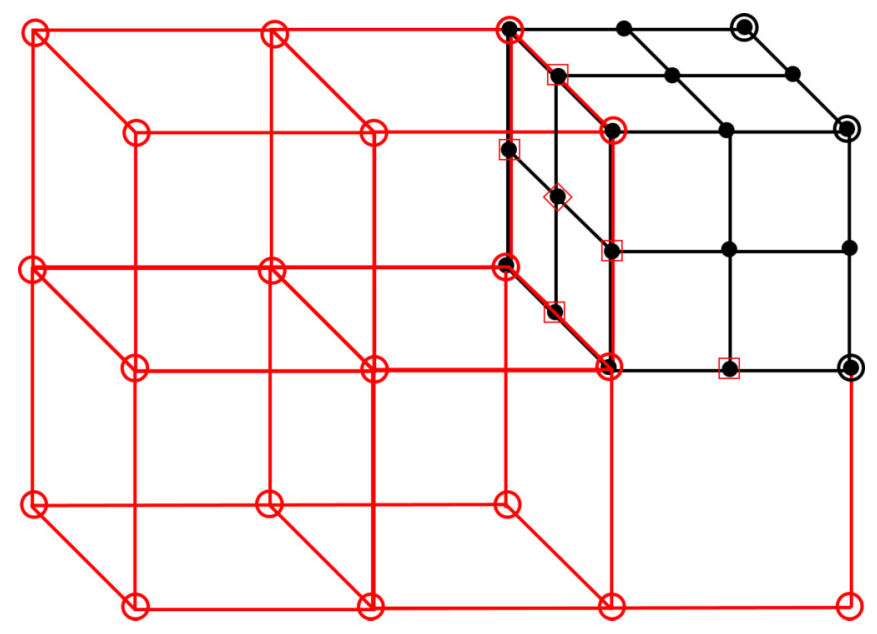

FIG. 1. Visualization of the grid arrangement around transition in resolution; the nodes in $\bigcirc$ are located on the coarse grid and the nodes in $\bullet$ are located on the fine grid. The nodes in $(\bullet+\bigcirc)$ are in collocated transition nodes, the nodes in $(\bullet+\square)$ are transition fine nodes located on the edge-center of coarse grid, and the nodes in $(\bullet+\diamond)$ are transition fine nodes located on the face-center of coarse grid.

A typical refinement interface between the coarse grid at $L$ th level and the fine grid at $(L+1)$ th level is illustrated in Fig. 1. Let us use subscript $c$ to denote variables defined on the coarse grid and $f$ for fine grid quantities. Thus, defining $\delta x_{c}$ and $\delta x_{f}$ the spatial grid spacing of the coarse and fine grids, respectively.

$$
\delta x_{f}=\frac{1}{2} \delta x_{f}=\frac{1}{2^{(L+1)}} \delta x_{0}, \delta t_{f}=\frac{1}{2} \delta t_{f}=\frac{1}{2^{(L+1)}} \delta t_{0},
$$

where $\delta x_{0}$ and $\delta t_{0}$ are mesh size and time step at the coarsest level $L=0$, respectively. Due to the uniform convective scaling, the nondimensional relaxation time and the second order Hermite coefficients $\left(\mathcal{A}_{\alpha \beta}^{(1)}\right)$ should be rescaled between the fine grid and the coarse grid following

$$
\frac{\tau_{f}-0.5}{\tau_{c}-0.5}=\frac{\delta x_{c}}{\delta x_{f}}=2, \frac{\mathcal{A}_{\alpha \beta, c}^{(1)}}{\mathcal{A}_{\alpha \beta, f}^{(1)}}=2 \frac{\overline{\tau_{c}}}{\overline{\tau_{f}}} .
$$

In the present multidomain approach, the collisionstreaming algorithm described in Eq. (4) cannot be applied at the transition nodes. A communication algorithm between the fine and coarse domains is needed to exchange information through interfaces. Compared with commonly used grid refinement techniques in athermal flows, some additional treatments are needed in the present case to solve the entropy equation and to compute the macroscopic variable gradients appearing in the Hermite coefficients and correcting terms involved in the compressible HRR-LB method. The procedure of the communication for the grid refinement on HRR-LB solver is described as follows:

(1) The collision step is done at all (fine and coarse) nodes using Eq. (5).

(2) The streaming step is applied at ordinary nodes $\bullet$ and which are far from the transition interface. Meanwhile, the propagation step is also applied on the coarse grid nodes located on the transition interface labeled by $(\bullet+\bigcirc)$.
(3) The moments including the macroscopic values ( $\rho$, $\left.u_{\alpha}, T\right)$ and the second order Hermite coefficients $\left(\mathcal{A}_{\alpha \beta}^{(1)}\right)$ are computed via Eq. (6) at ordinary nodes $\bullet$ and $\bigcirc$ and on the coarse grid points located on the transition $(\bullet+\bigcirc)$.

(4) The entropy is updated by solving Eq. (13) at ordinary nodes $\bullet$ and $\bigcirc$ and on the coarse grid points located at the transition $(\bullet+\bigcirc)$.

(5) Time interpolation is performed to evaluate the quantities $\left(\rho, u_{\alpha}, T, \mathcal{A}_{\alpha \beta}^{(1)}\right)$ on the fine grid points colocated with coarse grid ones on the transition interface marked by $(\bullet+$ ). The time interpolation method is described in the next paragraph.

(6) The gradients represented by a general form $\nabla \phi$ in the second order Hermite coefficients $\mathcal{A}_{\alpha \beta}^{(1, F D)}$ and in the correcting term $\psi$ are calculated at the fine grid nodes $\bullet$ which are one cell away from the transition interface.

(7) Spatial interpolation is used to update the moments $\left(\rho, u_{\alpha}, T, \mathcal{A}_{\alpha \beta}^{(1)}\right)$ at nodes $(\bullet+\square)$ and on nodes $(\bullet+\diamond)$. The detailed algorithm of 3D spatial interpolation is also described in the following paragraph.

(8) With updated macroscopic values, the gradients represented by a general form $\nabla \phi$ in the second order Hermite coefficients $\mathcal{A}_{\alpha \beta}^{(1, F D)}$ and in the correcting term $\psi$ are calculated at the ordinary nodes $\bullet, \bigcirc$ and on the coarse grid points located at the transition $(\bullet+\bigcirc)$.

(9) Time and space interpolations are used on the gradients $\nabla \phi$ to update the value of $\mathcal{A}_{\alpha \beta}^{(1, F D)}$ and correcting term $\psi$ on the fine grid points located at the transition $(\bullet+\square)$ and $(\bullet+\diamond)$

(10) After the above steps all the data required in Eq. (5) for ordinary fine nodes $\bullet$ are known and the second collision, streaming and moment steps are applied at all fine grid nodes.

(11) The entropy value of the second step on fine grid is update by solving Eq. (13) at the ordinary nodes $\bullet$ and $\bigcirc$.

(12) The Hermite coefficients $\mathcal{A}_{\alpha \beta}^{(1, F D)}$ and the correcting term $\psi$ are calculated on the fine grids nodes $\bullet$ and $(\bullet+\bigcirc)$.

(13) The Hermite coefficients $\left.\mathcal{A}_{\alpha \beta}^{(1)}\right)$ at nodes $(\bullet+\bigcirc)$ are duplicated and rescaled from coarse grid scale to fine grid scale using Eq. (15), then distribution functions at coarse grid nodes $(\bullet+\bigcirc)$ are assembled to prepare a pre-collisionstreaming distribution function for the coarse nodes at the transition nodes $(\bullet+\bigcirc)$ for the next time step.

(14) After the above steps all the data required in Eq. (5) for fine grid and coarse grid at same physical time are known for the next time step.

\section{B. Time interpolation algorithm}

As mentioned above, an interpolation step is needed at the transition interface. And the temporal interpolation and spatial interpolation are employed in the grid refinement procedure to communicate information of the distribution function and macroscopic variables, e.g., entropy $s$, temperature $T$. Here, the detailed algorithm of temporal and spatial interpolation is presented.

First, in temporal view of a spatially collocated nodes, the information on the fine grid and on the coarse grid at even time steps are synchronous, as shown in Fig. 2. However, the variables on the fine grid at odd time steps must be interpolated from the coarse grid. 



FIG. 2. Temporal interpolation along time line. The nodes in $(\bullet+\bigcirc)$ are in collocated transition nodes. (a) Odd time step, (b) even time step.

The overlapped $(\bullet+\bigcirc)$ nodes are in even time steps while the (• only) nodes are in odd time steps. The following second-order scheme is used in this study to interpolate macroscopic variables, $\rho, u_{\alpha}, T$, nonequilibrium moment $\mathcal{A}_{\alpha \beta}^{(1)}$ and correction term $\psi_{i}$, which is expressed in a generic variable $\phi$,

$$
\phi_{f}^{(t)}=\phi_{f}^{\left(t-\delta t_{f}\right)}+\frac{\phi_{c}^{\left(t+\delta t_{f}\right)}-\phi_{f}^{\left(t-2 \delta t_{f}\right)}}{3},
$$

where $\phi_{c}^{\left(t+\delta t_{f}\right)}$ is practically obtained in advance on coarse grid.

\section{Spatial interpolation technique in 3D grid refinement}

In three-dimensional grid refinement, two-step approach of spatial interpolation is performed to reduce the complexity of interpolation on surface. In the first step, to obtain the variables located on the fine grid points located in the transition, which is marked by $(\bullet+\square)$ in Fig. 3, a one-dimensional interpolation is performed along edges of interface which is parallel to the refinement interface.

For instance (type A nodes in Fig. 3, the 1D interpolation performed along the $x$-direction edge is given as follows:

$$
\begin{aligned}
\phi(i, j, k)= & -\frac{1}{16} \phi(i-3, j, k)+\frac{9}{16} \phi(i-1, j, k) \\
& +\frac{9}{16} \phi(i+1, j, k)-\frac{1}{16} \phi(i+3, j, k),
\end{aligned}
$$

where the cubic interpolation is adopted in the above equation since the linear interpolation can not conserve the mass [15]. In addition, an asymmetric scheme is adopted where the number of neighbors is not fulfilled in Eq. (17), type B nodes

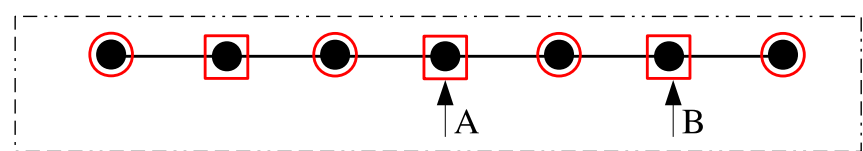

FIG. 3. 1D view along transition edge. The nodes in $(\bullet+\bigcirc)$ are in collocated transition nodes, the nodes in $(\bullet+\square)$ are transition fine nodes located on the edge-center of coarse grid.

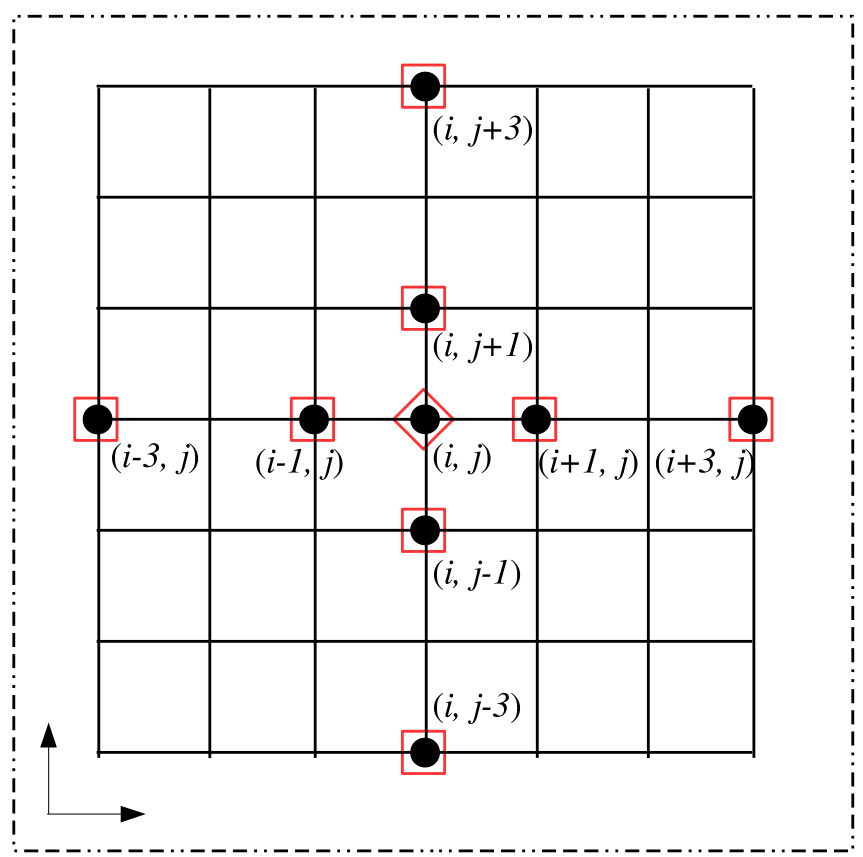

FIG. 4. 2D spatial interpolation in $x-y$ plane. The nodes in $(\bullet+$ $\square)$ are transition fine nodes located on the edge-center of coarse grid. $(\bullet+\diamond)$ are transition fine nodes located on the face-center of coarse grid.

in Fig. (1 b), the formula is given by the following:

$$
\begin{aligned}
\phi(i, j, k)= & -\frac{2}{16} \phi(i-3, j, k)+\frac{12}{16} \phi(i-1, j, k) \\
& +\frac{6}{16} \phi(i+1, j, k) .
\end{aligned}
$$

Then a two-dimensional interpolation is performed on the transition interface to update the variables located in center point of coarse grid. For instance, as shown in Fig. 4, the biinterpolation performed in $x-y$ plane is given as follows:

$$
\begin{aligned}
\phi(i, j, k)= & \frac{1}{2}\left[-\frac{1}{16} \phi(i-3, j, k)+\frac{9}{16} \phi(i-1, j, k)\right. \\
& \left.+\frac{9}{16} \phi(i+1, j, k)-\frac{1}{16} \phi(i+3, j, k)\right] \\
& +\frac{1}{2}\left[-\frac{1}{16} \phi(i, j-3, k)+\frac{9}{16} \phi(i, j-1, k)\right. \\
& \left.+\frac{9}{16} \phi(i, j+1, k)-\frac{1}{16} \phi(i, j+3, k)\right] .
\end{aligned}
$$

Similarly, when the required number of neighbors is not available, the same asymmetric scheme as in Eq. (18) is used in the associated direction.

\section{Grid refinement for entropy equation}

In the present compressible HRR-LBM, the entropy equation is solved by a finite volume method. Although the FVM is suitable for nonuniform grids, the same multidomain computational grid is used in the FVM to keep the consistency with the LB solver.

In this study, the mesh size $\delta_{x}$ and the time step $\delta_{t}$ in entropy equation are the same as those used to solve the 
LB equation in each domain. Similarly, the same spatial and temporal interpolation techniques are used on entropy $s$ in the FVM as those used in LB solver for macroscopic variables. For instance, the spatial interpolation is written as

$$
\begin{aligned}
s(i, j, k)= & -\frac{1}{16} s(i-3, j, k)+\frac{9}{16} s(i-1, j, k) \\
& +\frac{9}{16} s(i+1, j, k)-\frac{1}{16} s(i+3, j, k),
\end{aligned}
$$

while time interpolation is given by

$$
s_{f}^{(t)}=s_{f}^{\left(t-\delta t_{f}\right)}+\frac{s_{c}^{\left(t+\delta t_{f}\right)}-s_{f}^{\left(t-2 \delta t_{f}\right)}}{3} .
$$

\section{RESULTS AND DISCUSSION}

The proposed grid refinement method is now assessed considering a set of test cases with increasing level of difficulty.

\section{A. Isentropic vortex convection}

The first test case deals with the convection of an isentropic vortex by an inviscid uniform flow. The computational domain size was $[0,10] \times[0,10] \times[0,0.05]$. The free-stream conditions were $\rho_{\infty}=1, u_{\infty}=1.0, v_{\infty}=0, p_{\infty}=1, \mathrm{Ma}_{\infty}=$ 0.845 . At the initial time, the following disturbance was added to the above free-stream:

$$
\begin{gathered}
\rho=\left[1-\frac{(\gamma-1) b^{2}}{8 \gamma \pi^{2}} e^{1-r^{2}}\right]^{\frac{1}{\gamma-1}}, p=\rho^{\gamma}, \\
u=u_{\infty}-\frac{b}{2 \pi} e^{\frac{1}{2}\left(1-r^{2}\right)}\left(y-y_{c}\right), \\
v=v_{\infty}+\frac{b}{2 \pi} e^{\frac{1}{2}\left(1-r^{2}\right)}\left(x-x_{c}\right),
\end{gathered}
$$

where $b=0.5$ is the vortex strength and $r=\left[\left(x-x_{c}\right)^{2}+\right.$ $\left.\left(y-y_{c}\right)^{2}\right]^{1 / 2}$ is the distance from the vortex center $\left(x_{c}, y_{c}\right)=$ $(5,5)$.

The grid was refined in the subdomain defined as $[3,7] \times$ $[3,7] \times[0,0.05]$ with a mesh size $\Delta x_{\min }=0.025$. The grid size of the outer part of the computational domain was $\Delta x=2 \Delta x_{\min }$. In our simulation, the minimum time step of the simulation was $\Delta t_{\min }=0.005$. The maximum time step was $\Delta t_{\max }=0.01$. To simulate an inviscid flow, the dynamic viscosity was set equal to $\mu=10^{-15}$. Periodic boundary conditions are implemented in all directions.

Figures 5, 6, and 7 show the density, velocity, and temperature contours after 19T, respectively. Here, $1 \mathrm{~T}$ is 1 flowthrough-time, i.e., the time needed by the vortex to travel over a distance equal to the computational domain size. In the figures, the grid refinement region is colored with gray. It can be very clearly seen that there is no oscillation or discontinuity occurring on the interface between the fine mesh and coarse mesh. The shape of the initial perturbations are very well preserved even after 19T. After 20 flow-through-time (FTT), the distributions of density, velocity and temperature along the domain symmetry lines are displayed in Fig. 8. The results denoted by blue line are obtained by present LB without grid refinement on a grid with the same mesh as the fine subdomain. It is observed that both the results with and without grid refinement are very close to the exact solution. The $L_{2}$ errors obtained using different $\Delta x_{\min }$ are shown in
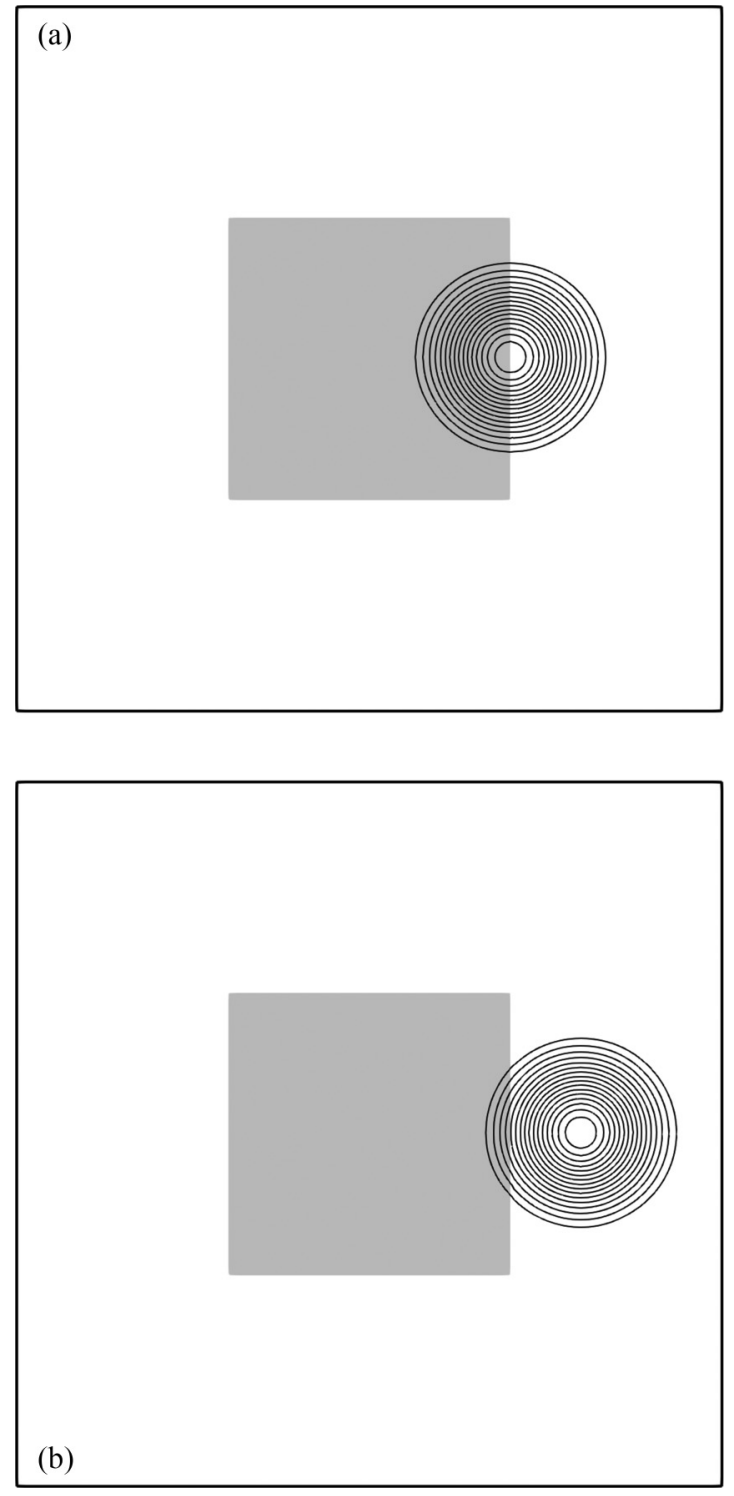

FIG. 5. Computed density field at different time of 19.2T (a) and 19.3T (b). The grid-refined subdomain is marked by the square in gray.

Fig. 9. It can be found that the present grid-refinement method performed a second order convergence rate.

\section{B. Shock-vortex interaction}

With the shock and vortex moving across the interface between the fine mesh and coarse mesh, the shock-vortex interaction problem is a very relevant test case to validate the grid refinement method. In this problem, a single vortex moves from right to left and interact with a stationary normal shock wave. The right and left states of the normal shock are as follows:

$$
\begin{gathered}
\rho_{R}=1.0, \quad \rho_{L}=\frac{(\gamma+1) M_{s}^{2}}{2+(\gamma-1) M_{s}^{2}} \rho_{R}, \\
p_{R}=1.0, \quad p_{L}=\left(\frac{2 \gamma}{\gamma+1} M_{s}^{2}-\frac{\gamma-1}{\gamma+1}\right) p_{R},
\end{gathered}
$$



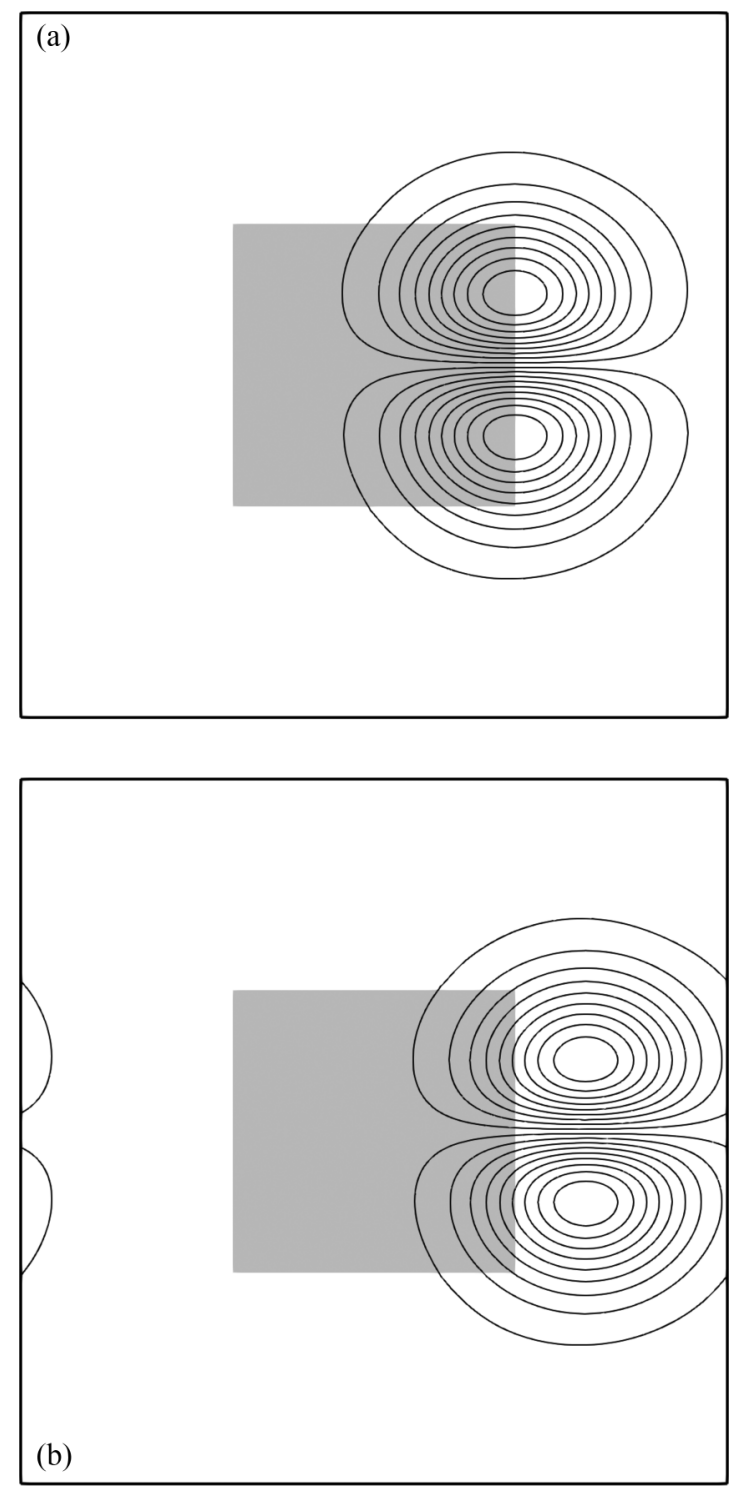

FIG. 6. Computed streamwise velocity ( $x$-direction) field at different time of 19.2T (a) and 19.3T (b). The grid-refined subdomain is marked by the square in gray.

$$
\begin{gathered}
T_{R}=p_{R} / \rho_{R}, \quad T_{L}=p_{L} / \rho_{L}, \\
u_{R}=-M_{s}, \quad u_{L}=-\frac{2+(\gamma-1) M_{s}^{2}}{(\gamma+1) M_{s}^{2}} M_{s}, \\
v_{R}=0, \quad v_{L}=0 .
\end{gathered}
$$

The initial density, pressure, tangential and radial velocity fields of the vortex are given by

$$
\begin{gathered}
\rho_{\theta}(r)=\left[1-\frac{\gamma-1}{2} M_{v}^{2} r \exp \left(1-r^{2}\right)\right]^{\frac{1}{\gamma-1}}, \\
p(r)=\frac{1}{\gamma} \rho^{\gamma}(r), \\
u_{\theta}(r)=M_{v} r \exp \left[\left(1-r^{2}\right) / 2\right], u_{r}(r)=0,
\end{gathered}
$$

where the distance from the vortex core $r$ is nondimensionalized by the vortex radius $R$. The above flow field of vortex is
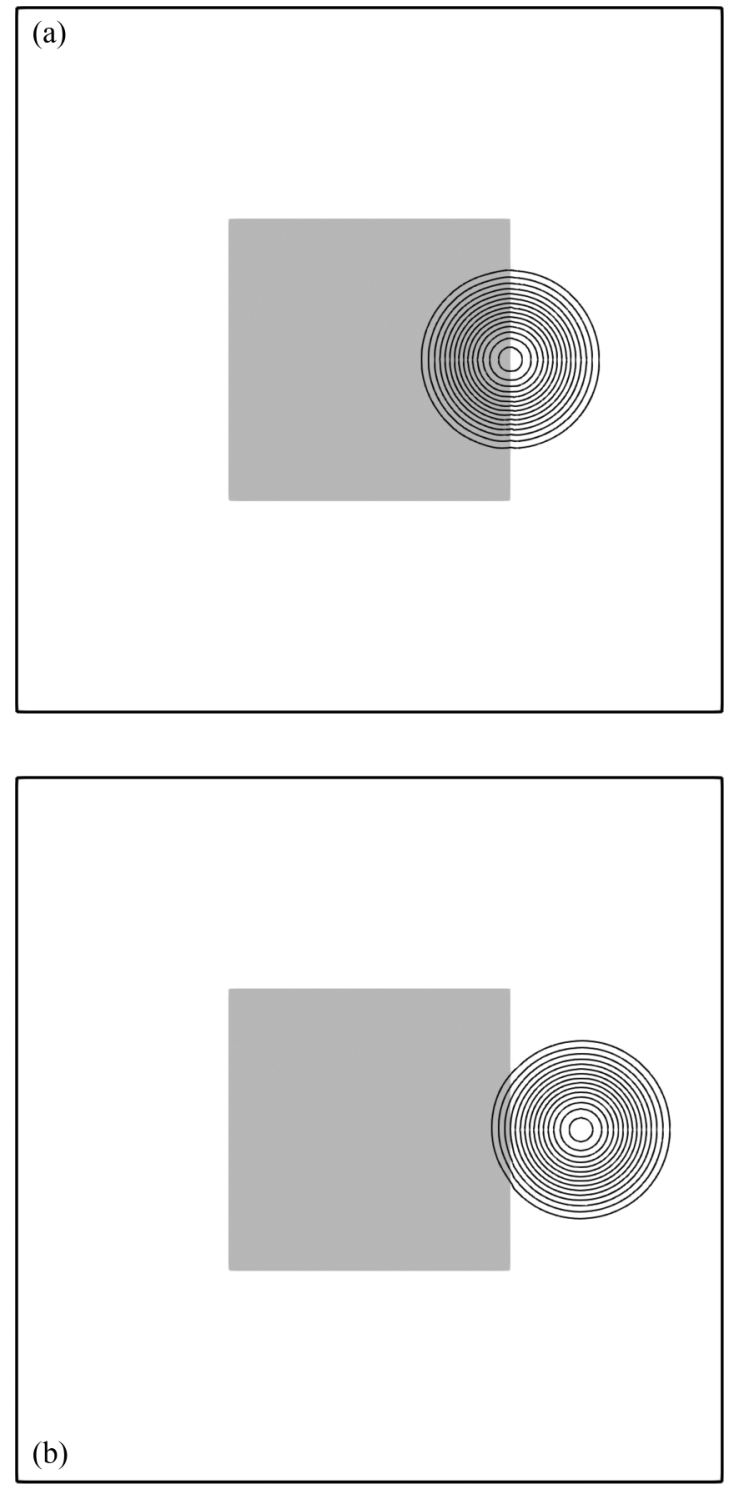

FIG. 7. Computed temperature field at different time of 19.2T (a) and $19.3 \mathrm{~T}$ (b). The grid-refined subdomain is marked by the square in gray.

added to the upstream of the shock wave at initial time. The following flow parameters were used in the test:

$$
M_{s}=1.2, \quad M_{v}=0.25, \operatorname{Re}=800, R=1, \gamma=1.4 .
$$

The Reynolds number was defined by $\operatorname{Re}=\rho_{R} a_{R} R / \mu_{R}$ with $a_{R}$ being the sound speed of the upstream of the shock. A computational domain $[-20 R, 8 R] \times[-12 R, 12 R] \times$ $\left[0, \Delta x_{\max }\right]$ was considered in the simulation. Here, $\Delta x_{\max }$ was the maximum mesh size of the grid system. Initially, the vortex was located at $x=2 R$ and $y=0$, and the planar shock wave was specified at $x=0$ by imposing density, velocity, and pressure variables corresponding to the above left and right states of the normal shock. In the computational domain, a subdomain with grid refinement was introduced. The minimum (in the fine grid subdomain) and maximum (in the outer subdomain) grid size were $\Delta x_{\min }=0.0125 R, \Delta x_{\max }=$ $0.025 R$. Two grid-refinement configurations denoted by 

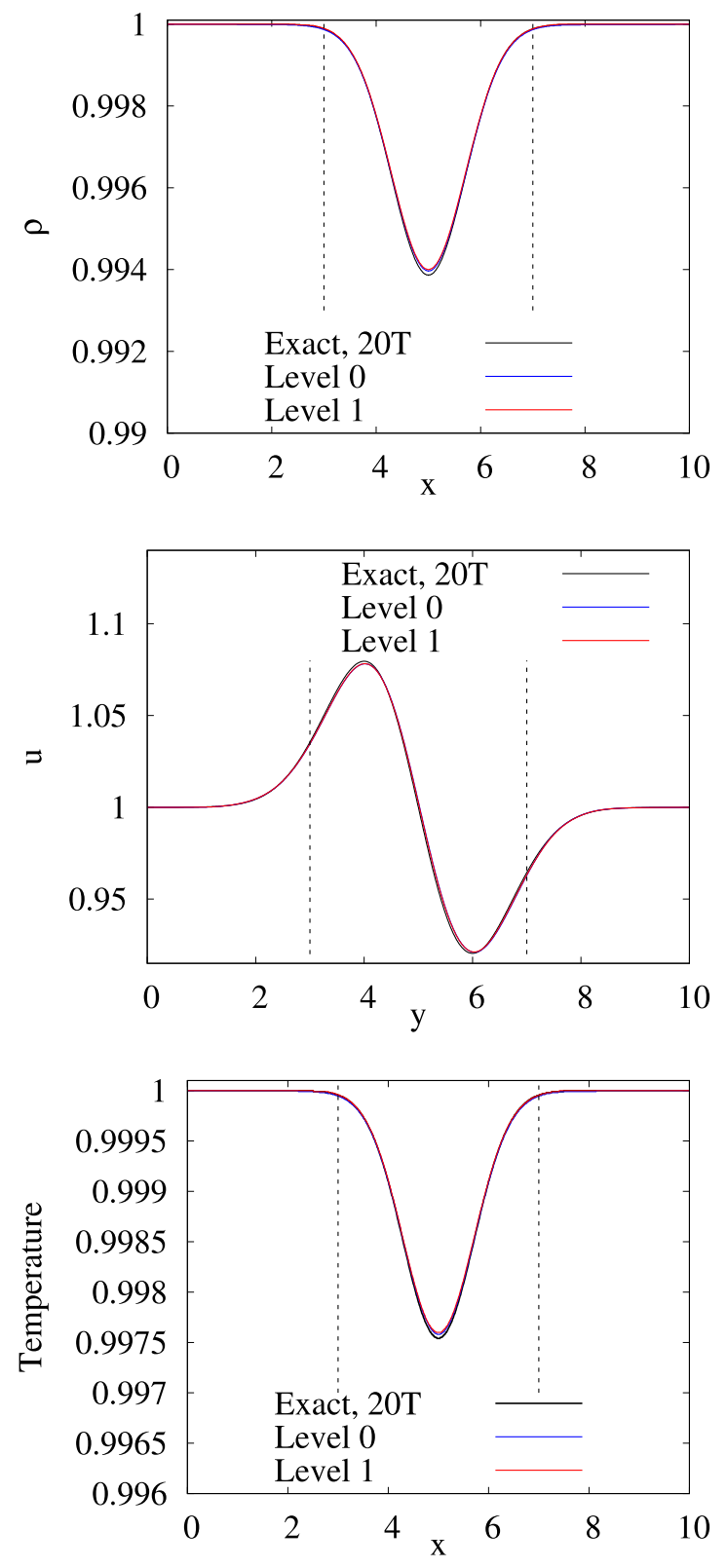

FIG. 8. Distributions of density, velocity, and temperature at midline at $t=20 \mathrm{~T}$. The refined domain is located between the two dotted lines.



FIG. 9. L2 error obtained by the LBM with the present grid refinement approach. $N=10 / \Delta x_{\min }$.
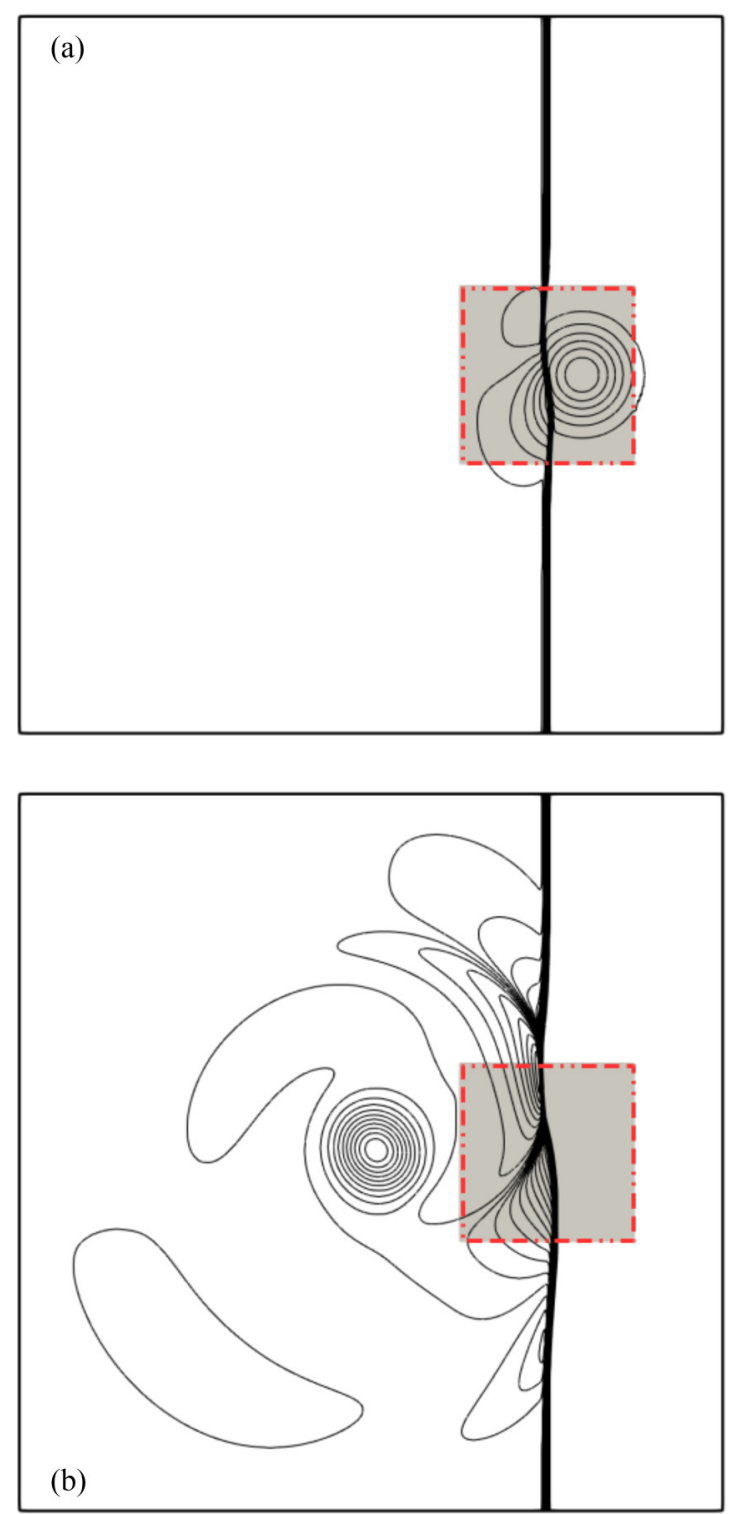

FIG. 10. Sound pressure field obtained by the present HRR-LBM with grid refinement on Grid-a configuration at $t=1$ (a), and $t=6$ (b), respectively. The grid-refined domain is inside the square marked by red dotted line.

Grid-a and Grid-b were considered to assess the robustness and the accuracy of the proposed grid refinement method. For Grid-a configuration, the grid refinement was implemented in the area $[-2 R, 2 R] \times[-2 R, 2 R] \times\left[0, \Delta x_{\max }\right]$. Thus, the initial center of the vortex was at the interface between fine and coarse mesh in Grid-a. The grid refinement region of Grid-b configuration was $[-0.5 R, 0] \times[-R, 1.5 R] \times$ $\left[0, \Delta x_{\max }\right]$. It can be found that the normal shock was initially located at the fine-coarse mesh interface in Grid-b.

The sound pressure fields obtained on Grid-a and Grid-b are plotted in Figs. 10 and 11, respectively. Here, the sound pressure $\Delta p$ is defined as $\Delta p=\left(p-p_{L}\right) / p_{L}$. In these two figures, the grid refinement regions are also colored with gray. It is observed that the fields computed in both configurations are very similar to the reference solution given in Ref. [58]. There is no oscillation or discontinuity found on the 

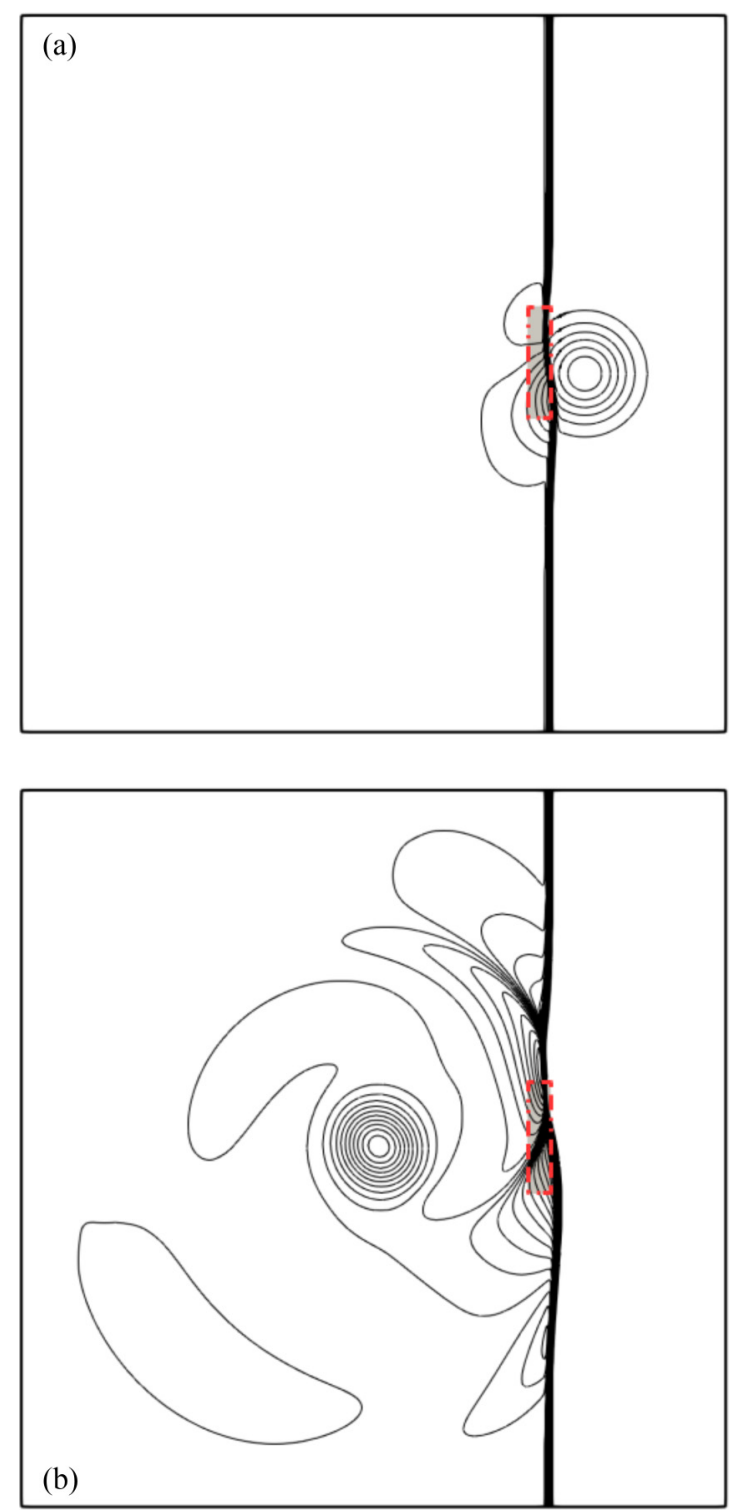

FIG. 11. Sound pressure field obtained by the present HRR-LBM with grid refinement on Grid-b configuration at $t=1$ (a), and $t=6$ (b), respectively. The grid-refined domain is inside the square marked by red dotted line.

coarse-fine mesh interface in both figures. For the quantitative comparison, the distributions of the sound pressure are plotted in Fig. 12, in which $r$ is the distance from the center of the vortex. The results represented by symbols are related to the reference solution given in Ref. [58]. It is seen that the results obtained on both Grid-a and Grid-b are in very good agreement with the reference results.

To further validate the method of grid refinement, the shock-vortex interaction with flow parameters $M_{s}=$ $1.2, M_{v}=0.5, \operatorname{Re}=400$ is also simulated in this study. Figure 13 presents the density field at $t=8$. The contour levels from 0.92 to 1.55 with an increment of 0.0053 are used, which are the same with Fig. 10 in Ref. [58]. It is observed that the density contours obtained by the LB-gridrefinement smoothly through the refinement interfaces and perfectly match the reference results.
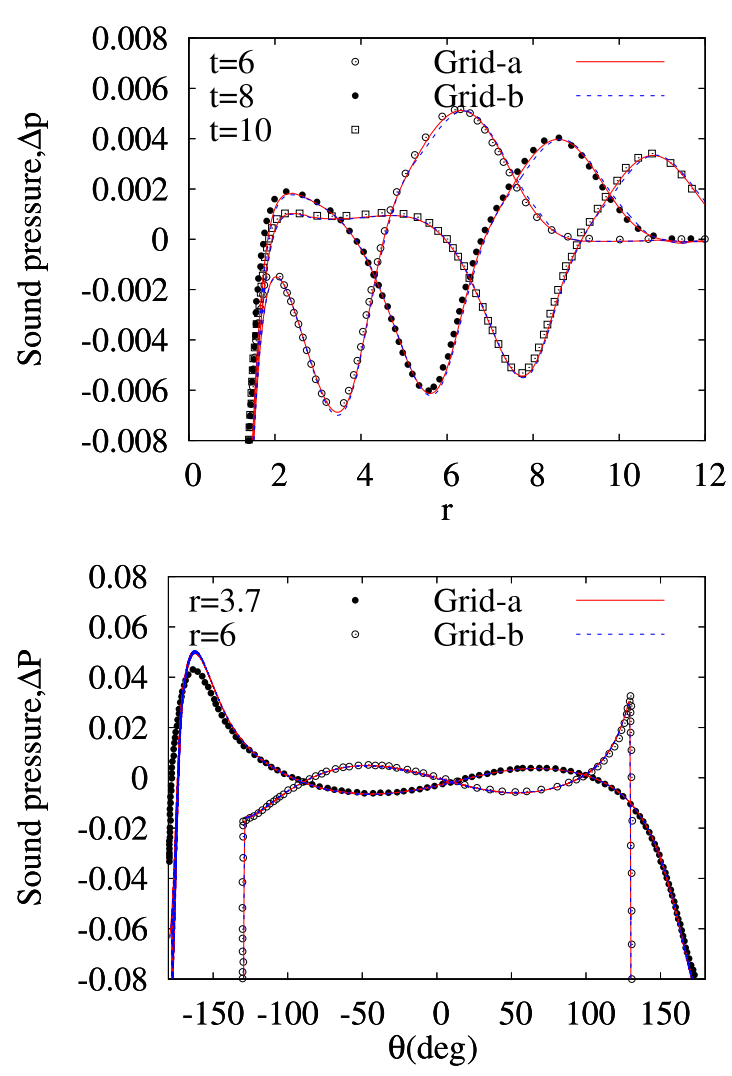

FIG. 12. Distributions of the sound pressure. top: radial distribution at the angle $\theta=-45^{\circ}$; bottom: circumferential distribution at $t=6$. The reference data in Ref. [58] are denoted by symbols.

\section{Transonic viscous flow over NACA0012 airfoil}

Next, a viscous flow over the NACA0012 airfoil at $M a_{\infty}=0.85$ was investigated using the grid-refinement technique. The computational domain size was $x \in[-12,22] \times$ $y \in[-12,12] \times z \in[0,0.2]$, where the chord of the airfoil was chosen as a reference length scale. On the left boundary of the domain, the subsonic inflow parameters were $\mathrm{Ma}_{\infty}=0.85, \alpha=0, \rho_{\infty}=1, p_{\infty}=1 / \gamma, u_{\infty}=$ $0.85, v_{\infty}=0, \operatorname{Re}=10000$. The isothermal nonslip boundary condition was implemented on the surface of the airfoil.



FIG. 13. Density field at $t=8, M_{v}=0.5, \mathrm{Re}=400$. The contour levels are from 0.92 to 1.55 with an increment of 0.0053 . 



(b)

FIG. 14. Instantaneous Ma number (a) and vorticity fields (b) at time $t=16.36$. Grid refinement interfaces are denoted by solid lines.

The top, bottom, and right boundary were subsonic outlet conditions.

A computational grid with a seven-level grid refinement was used in the simulation. To capture the geometry of the airfoil with high accuracy, the minimum grid size $\Delta x_{\min }=$ 0.0015625 was used near the solid wall. In this unsteady



FIG. 15. Pressure coefficients on the NACA0012 airfoil at $t=16.36$.

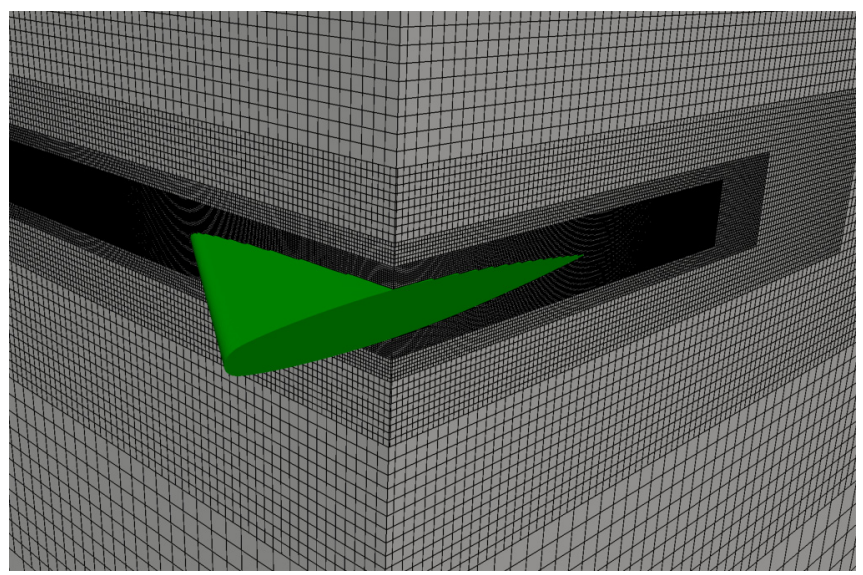

FIG. 16. Sketch of grid refinement for inviscid flows past ONERA-M6. There are approximately 800 nodes in chord length at the finest level.

test case, the complex flow field is formed by the shear layer development (boundary layers, wake), vortex shedding (wake), and sound waves radiation and boundary layer/shock wave interaction. Figure 14 shows the instantaneous Mach number and vorticity fields at time $t=16.36$. As can be seen from the figure, there is no nonphysical oscillation rising at the coarse-fine grid interfaces. For a quantitative comparison, the instantaneous pressure distribution along the surface of the airfoil at time $t=16.36$ is compared with reference DNS data [59] in Fig. 15. A very good agreement can be found, showing the accuracy of the grid refinement technique.

\section{Inviscid transonic flow over ONERA M6 wing}

At last, we validated the grid refinement technique on a three-dimensional aerodynamic flow, namely, the transonic inviscid flow around the ONERA M6 wing. The free-stream Mach number was taken equal to $\mathrm{Ma}=0.8397$ and the angle of attack was set to $3.06^{\circ}$. In the simulation, the computational domain was $[-12.0,22.0] \times[0.0,12.0] \times[-5.0,5.0]$. The sketch and set up of the grid refinement are displayed in Fig. 16 and Table I. At the inflow boundary the variables $(u, v, w, p)$ were given by freestream values $\left(u_{\infty}, v_{\infty}, p_{\infty}\right)$, at the outflow boundary condition with prescribed outlet pressure $p_{\infty}$ was implemented. On the wall, a free-slip adiabatic condition was enforced to recover the inviscid flow solution.

TABLE I. Setup of grid refinement for transonic flow over ONERA M6 wing.

\begin{tabular}{ccccc}
\hline \hline $\mathrm{L}$ & Start position & End position & $\Delta x$ & $\Delta t$ \\
\hline 0 & $(-12.0,0.0,-5.0)$ & $(22.0,12.0,5.0)$ & 0.1 & 0.03333333 \\
1 & $(-3.0,0.0,-1.0)$ & $(5.0,5.0,1.0)$ & 0.05 & 0.01666667 \\
2 & $(-2.0,0.0,-0.5)$ & $(3.0,3.0,0.5)$ & 0.025 & 0.00833333 \\
3 & $(-1.0,0.0,-0.2)$ & $(2.0,2.0,0.2)$ & 0.0125 & 0.00416667 \\
4 & $(-0.3,0.0,-0.1)$ & $(1.5,1.5,0.1)$ & 0.00625 & 0.00208333 \\
5 & $(-0.1,0.0,-0.07)$ & $(1.3,1.3,0.07)$ & 0.003125 & 0.00104167 \\
6 & $(-0.05,0.0,-0.05)$ & $(1.25,1.25,0.05)$ & 0.0015625 & 0.00052083 \\
\hline \hline
\end{tabular}




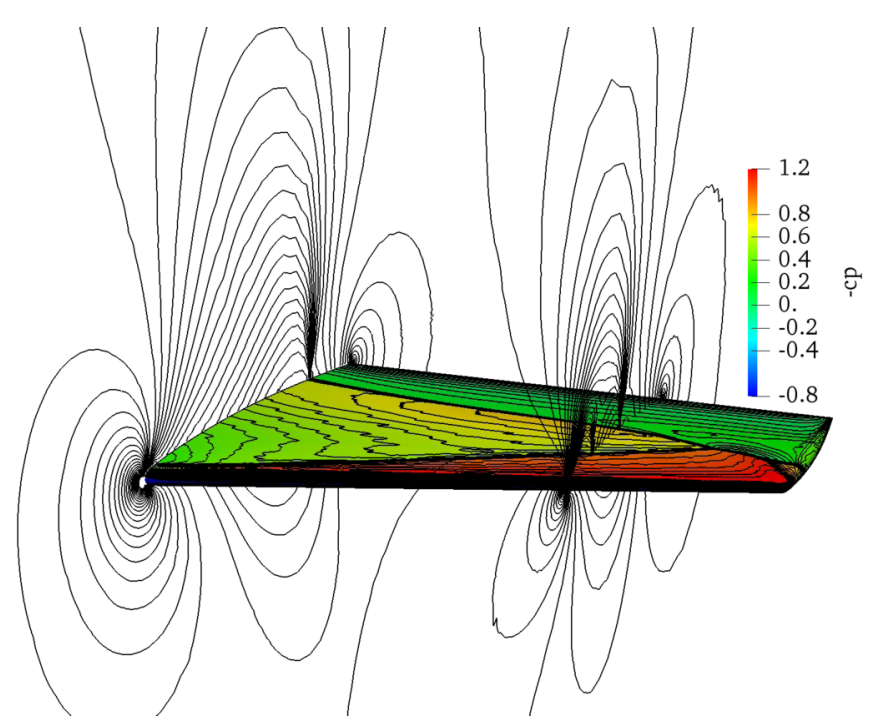

FIG. 17. Pressure coefficient obtained by the present LB with grid refinement for inviscid flows past ONERA-M6.

Figure 17 shows the pressure coefficient obtained by the present LB with grid refinement. It can be observed that the typical $\lambda$-shock topology is very well captured. The contours of pressure coefficient on symmetry wall is also presented in Fig. 17. It can observed that the pressure contours obtained smoothly cross the interface of grid refinement.

To further validate the accuracy of the LBM with 3D grid refinement, the values of pressure coefficient at different locations of the wing are plotted in Fig. 18. The excellent agreement between the present results and reference results can be found. The reference solution is calculated by DG method for inviscid compressible flows in Ref. [60]. The experimental results were data obtained by wind tunnel for turbulent viscous flows in Ref. [61]. In addition, the drag and lift coefficients obtained by the present method is respectively $C_{d}=0.014$ and $C_{l}=0.279$ which are very close to the reference data $C_{d}=0.013 \sim 0.0136$ and $C_{l}=0.278 \sim 0.29$ given in Refs. $[60,62]$.

\section{CONCLUSIONS}

A three-dimensional grid refinement method within the framework of hybrid recursive regularized lattice Boltzmann method was developed in this paper. The numerical tests including isentropic vortex propagating through transition interface, shock-vortex interaction with intersection between grid refinement interface and shock corrugation, and transonic flows over 3D ONERA M6 wing with seven embedded grid levels were performed to assess the proposed implementation. The numerical validation shows the excellent performance of the proposed implementation for 3D grid refinement in the applications of compressible aerodynamics with and without shocks.
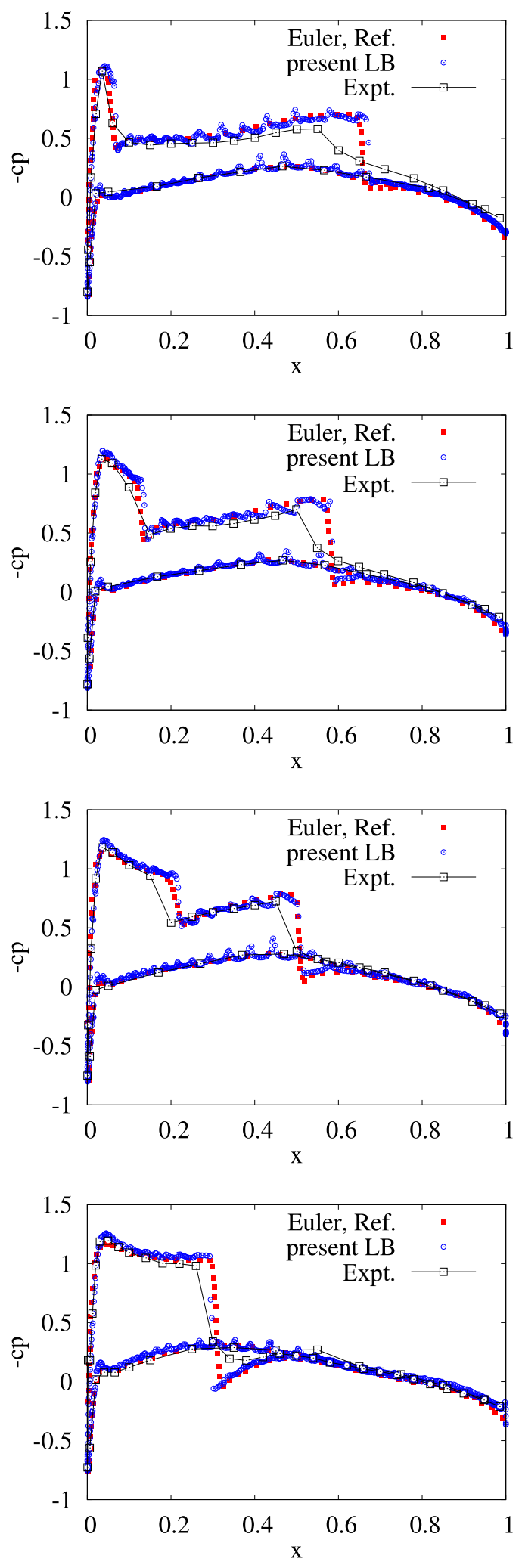

FIG. 18. Pressure coefficient at $y=20 \%$ span, $y=44 \%$ span, $y=65 \%$ span, and $y=90 \%$ span (from top to bottom) obtained by the present LB with grid refinement for inviscid flows past ONERA-M6. 


\section{ACKNOWLEDGMENTS}

This work was granted access to the HPC resources of Aix-Marseille Université financed by the project Equip@Meso (ANR-10-EQPX-29-01) of the program
Investissements d'Avenir supervised by the Agence Nationale de la Recherche. This work was performed using HPC resources from GENCI-TGCC/CINES (Grant No. 2018-A0052A07679). This work was also supported by the ProLB software project [63].
[1] A. A. Mohamad, Lattice Boltzmann Method: Fundamentals and Engineering Applications with Computer Codes (Springer Science \& Business Media, Berlin, 2011).

[2] Z. Guo and C. Shu, Lattice Boltzmann Method and Its Applications in Engineering, Vol. 3 (World Scientific, Singapore, 2013).

[3] T. Krüger, H. Kusumaatmaja, A. Kuzmin, O. Shardt, G. Silva, and E. M. Viggen, The Lattice Boltzmann Method (Springer International Publishing, Switzerland, 2017).

[4] Y. Qian, D. D’Humires, and P. Lallemand, Europhys. Lett. 17, 479 (1992).

[5] S. Y. Chen and G. D. Doolen, Annu. Rev. Fluid Mech. 30, 329 (1998).

[6] I. Cheylan, G. Fritz, D. Ricot, and P. Sagaut, AIAA J. 57, 2758 (2019).

[7] L. Hao, K. Moriyama, W. Gu, and C.-Y. Wang, J. Electrochem. Soc. 163, F744 (2016).

[8] N. H. Ahmad, A. Inagaki, M. Kanda, N. Onodera, and T. Aoki, Boundary-Layer Meteorol. 163, 447 (2017).

[9] J. Jacob and P. Sagaut, Build. Environ. 139, 110 (2018).

[10] Y. Feng, P. Boivin, J. Jacob, and P. Sagaut, Phys. Rev. E 100, 023304 (2019).

[11] Z. Liu, X. Chu, X. Lv, H. Meng, S. Shi, W. Han, J. Xu, H. Fu, and G. Yang, in Proceedings of the 2019 IEEE International Parallel and Distributed Processing Symposium (IPDPS'19) (IEEE, Piscataway, NJ, 2019), pp. 557-566.

[12] C. Godenschwager, F. Schornbaum, M. Bauer, H. Köstler, and U. Rüde, in Proceedings of the International Conference on High Performance Computing, Networking, Storage and Analysis (SC'13) (IEEE, Piscataway, NJ, 2013), pp. 1-12.

[13] F. Schornbaum and U. Rude, SIAM J. Sci. Comput. 38, C96 (2016).

[14] O. Filippova and D. Hänel, J. Comput. Phys. 147, 219 (1998).

[15] D. Lagrava, O. Malaspinas, J. Latt, and B. Chopard, J. Comput. Phys. 231, 4808 (2012)

[16] A. Dupuis and B. Chopard, Phys. Rev. E 67, 066707 (2003).

[17] M. Rohde, D. Kandhai, J. Derksen, and H. E. Van den Akker, Int. J. Numer. Methods Fluids 51, 439 (2006).

[18] S. Geller, M. Krafczyk, J. Tölke, S. Turek, and J. Hron, Comput. Fluids 35, 888 (2006).

[19] K. N. Premnath, M. J. Pattison, and S. Banerjee, Physica A: Stat. Mech. Appl. 388, 2640 (2009).

[20] C. Janssen and M. Krafczyk, Comput. Math. Appl. 59, 2215 (2010).

[21] M. Hasert, K. Masilamani, S. Zimny, H. Klimach, J. Qi, J. Bernsdorf, and S. Roller, J. Comput. Sci. 5, 784 (2014).

[22] H. Chen, O. Filippova, J. Hoch, K. Molvig, R. Shock, C. Teixeira, and R. Zhang, Physica A: Stat. Mech. Appl. 362, 158 (2006).
[23] F. Gendre, D. Ricot, G. Fritz, and P. Sagaut, Phys. Rev. E 96, 023311 (2017).

[24] X. Zhang, J. Kang, Z. Guo, S. Xiong, and Q. Han, Comput. Phys. Commun. 223, 18 (2018).

[25] D. Yu, R. Mei, and W. Shyy, Int. J. Numer. Methods Fluids 39, 99 (2002).

[26] J. Tölke, S. Freudiger, and M. Krafczyk, Comput. Fluids 35, 820 (2006).

[27] H. Touil, D. Ricot, and E. Lévêque, J. Comput. Phys. 256, 220 (2014).

[28] A. Fakhari and T. Lee, Phys. Rev. E 89, 033310 (2014).

[29] Y. Kuwata and K. Suga, J. Comput. Phys. 311, 348 (2016).

[30] G. Eitel-Amor, M. Meinke, and W. Schröder, Comput. Fluids 75, 127 (2013).

[31] M. Geier, M. Schönherr, A. Pasquali, and M. Krafczyk, Comput. Math. Appl. 70, 507 (2015).

[32] M. Geier, A. Greiner, and J. G. Korvink, Eur. Phys. J.: Spec. Top. 171, 173 (2009).

[33] H. Liu, J. G. Zhou, and R. Burrows, Comput. Fluids 38, 1108 (2009).

[34] S. Wilhelm, J. Jacob, and P. Sagaut, Phys. Fluids 30, 065111 (2018).

[35] J. Latt and B. Chopard, Math. Comput. Simul. 72, 165 (2006).

[36] X. Shan, Phys. Rev. E 100, 043308 (2019).

[37] A. Kramer, D. Wilde, K. Kullmer, D. Reith, and H. Foysi, Phys. Rev. E 100, 023302 (2019).

[38] C. Coreixas, B. Chopard, and J. Latt, Phys. Rev. E 100, 033305 (2019).

[39] C. Coreixas, G. Wissocq, G. Puigt, J. F. Boussuge, and P. Sagaut, Phys. Rev. E 96, 033306 (2017).

[40] S. A. Hosseini, C. Coreixas, N. Darabiha, and D. Thévenin, Phys. Rev. E 99, 063305 (2019).

[41] X. W. Shan, X. F. Yuan, and H. D. Chen, J. Fluid Mech. 550, 413 (2006).

[42] N. Frapolli, S. S. Chikatamarla, and I. V. Karlin, Phys. Rev. E 92, 061301(R) (2015).

[43] P. Philippi, D. Siebert, L. Hegele Jr., and K. Mattila, J. Braz. Soc. Mech. Sci. Eng. 38, 1401 (2016).

[44] B. Dorschner, N. Frapolli, S. S. Chikatamarla, and I. V. Karlin, Phys. Rev. E 94, 053311 (2016).

[45] N. I. Prasianakis and I. V. Karlin, Phys. Rev. E 78, 016704 (2008).

[46] Y. Feng, P. Boivin, J. Jacob, and P. Sagaut, J. Comput. Phys. 394, 82 (2019).

[47] M. H. Saadat, F. Bösch, and I. V. Karlin, Phys. Rev. E 99, 013306 (2019).

[48] F. Renard, J. Boussuge, Y. Feng, and P. Sagaut (unpublished).

[49] S. Guo, Y. Feng, J. Jacob, and P. Sagaut, J. Comput. Phys. (to be published). 
[50] D. N. Siebert, L. A. Hegele Jr., and P. C. Philippi, Phys. Rev. E 77, 026707 (2008).

[51] O. Malaspinas, arXiv:1505.06900.

[52] P. J. Dellar, Comput. Math. Appl. 65, 129 (2013).

[53] G. Wissocq, P. Sagaut, and J. Boussuge, J. Comput. Phys. 380, 311 (2019).

[54] K. H. Kim, C. Kim, and O.-H. Rho, J. Comput. Phys. 174, 38 (2001).

[55] C. Hirsch, Numerical Computation of Internal and External Flows: The Fundamentals of Computational Fluid Dynamics (Elsevier, Amsterdam, 2007).

[56] A. Jameson, AIAA J. 55, 1487 (2017).
[57] Y. Feng, S. Guo, J. Jacob, and P. Sagaut, Phys. Fluids 31, 126103 (2019).

[58] O. Inoue and Y. Hattori, J. Fluid Mech. 380, 81 (1999).

[59] M. O. Bristeau, Numerical Simulation of Compressible NavierStokes Flows: A GAMM Workshop, Vol. 18 (Springer Science \& Business Media, Berlin, 2013).

[60] J. J. van der Vegt and H. Van der Ven, J. Comput. Phys. 141, 46 (1998).

[61] V. Schmitt, AGARD AR-138 (Report of the Fluid Dynamics Panel Working Group 04, 1979), https://www.scribd.com/ document/424255166/AGARD-AR-138-pdf.

[62] C. Koeck, Int. J. Numer. Methods Fluids 5, 483 (1985).

[63] http://www.prolb-cfd.com. 